\title{
QUALIDADE DA SUPERFÍCIE USINADA EM PISOS DE MADEIRA DE Corymbia E Eucalyptus
}

\author{
Francisco Altobelly Viana da Silva ${ }^{1}$, José Reinaldo Moreira da Silva ${ }^{1}$, Jordão Cabral Moulin ${ }^{1 *}$, \\ João Rodrigo Coimbra Nobre ${ }^{1}$, Anna Carolina de Almeida Andrade', Jonnys Paz Castro ${ }^{1}$
}

\begin{abstract}
1Universidade Federal de Lavras, Departamento de Ciência e Tecnologia da Madeira, Lavras, Minas Gerais - altobellyviana @ hotmail.com; jreinaldoms@gmail.com; jordao_cm@hotmail.com*; sokonobre@hotmail.com; carol_bertges@hotmail.com; jonnys_33@hotmail.com
\end{abstract}

Recebido para publicação: 14/11/2015 - Aceito para publicação: 13/06/2016

\section{Resumo}

O objetivo do trabalho foi gerar conhecimento científico que contribua para a melhor eficiência dos parâmetros envolvidos na usinagem a partir de diferentes métodos para qualificação da superfície usinada e avaliar o uso das espécies Eucalyptus e Corymbia na produção de piso, como também analisar a relação da densidade e desvio da grã com a rugosidade da madeira. Foram utilizadas as espécies E. dunnii, E. urophylla, E. grandis, $C$. citriodora e $C$. maculata, todas com 37 anos. Para o ensaio de usinagem, foram utilizados corpos de prova com dimensões de $800 \times 100 \times 25 \mathrm{~mm}$, velocidades de avanço de 15 e $30 \mathrm{~m}^{*} \mathrm{~min}^{-1} \mathrm{e}$ velocidade de corte da ferramenta de 18 e $25 \mathrm{~m}^{*} \mathrm{~s}^{-1}$. Foi realizada a qualificação da superfície usinada por meio do avanço por dente, análise visual e parâmetros de rugosidade, sendo realizada também a relação da densidade e desvio da grã com a rugosidade da madeira. As madeiras de todas as espécies foram classificadas como ruim e muito ruim pela qualificação visual, e os valores de avanço por dente foram acima dos indicados para pisos de madeira. Os menores valores dos parâmetros de rugosidade foram obtidos nas madeiras com maior densidade e menor desvio da grã. Ao utilizar os diferentes métodos de qualificação de superfícies nas madeiras de Eucalyptus e Corymbia, observou-se baixo potencial para utilizar essas madeiras em pisos. No entanto, é necessário que sejam realizados outros estudos, com diferentes velocidades de avanço e de corte, para verificar a possibilidade de melhoria nas qualidades superficiais dessas madeiras.

Palavras-chave: Usinagem de madeira; rugosidade; avanço por dente.

\begin{abstract}
Quality machined surface in woods floors Corymbia and Eucalyptus. The objective was to generate scientific knowledge that contributes to better efficiency of the parameters involved in machining from different evaluation methods of qualifying the machined surface and evaluate the use of species of Eucalyptus and Corymbia in floor production, but also to analyze the relationship of density and deviation of the grain with the roughness of wood. The species used were E. dunnii, E. urophylla, E. grandis, C. citriodora and C. maculata, all aged 37. For machining test were used test specimens with dimensions of $800 \times 100 \times 25 \mathrm{~mm}$, advance speeds 15 and $30 \mathrm{~m}^{*} \mathrm{~min}^{-1}$ and cutting speed of the tool 18 and $25 \mathrm{~m}^{*} \mathrm{~s}^{-1}$. It was held the qualification of the machined surface through the feed per tooth, visual analysis and roughness parameters, and also carried the relationship of the density and diversion of grain with roughness of wood. The wood of all species were classified as bad and very bad for the visual qualification, per tooth feed values were above recommended for wood floors. The lowest values of roughness parameters were obtained for the woods with higher density and lower deviation of the grain. By using different methods of qualification surfaces in Eucalyptus and Corymbia woods, low potential was observed to use these wood floors. However, it must be conducted other studies with different speeds of advance and cut to verify the possibility of improving the surface qualities of the wood.

Keyword: Wood machining; roughness; feed per tooth.
\end{abstract}

\section{INTRODUÇÃO}

O Brasil é um país com grande potencialidade no setor de base florestal, uma vez que sua atividade se divide em vários segmentos, como celulose e papel, carvão vegetal, móveis e madeira processada mecanicamente, que engloba a produção de madeira serrada, painéis reconstituídos, compensados, laminados e pisos.

A indústria de pisos de madeira no Brasil destaca-se no mercado mundial por estar entre os principais produtores. Houve aumento de $15 \%$ na produção durante o período de 2000 a 2010, passando de 20 a 23 milhões de $\mathrm{m}^{2}$ de pisos por ano. Porém houve uma queda na produção entre 2006 e 2009 , devido à crise econômica mundial. No ano de 2009, o Brasil foi responsável por $9 \%$ da exportação mundial, ficando atrás somente da China, responsável por 15\% (ASSOCIAÇÃO NACIONAL DOS PRODUTORES DE PISOS DE MADEIRA (ANPM), 2013). 
De acordo com características como dimensões, espécies, acabamento superficial e presença ou ausência de encaixes laterais, os pisos de madeira recebem nomenclaturas diferenciadas. Eles podem ser laminados, engenheirados ou maciços (ANPM, 2013).

As operações de processamento mecânico da madeira correspondem às condições de usinagem. Assim, para um produto com melhor acabamento, as operações de processamento devem ser monitoradas e avaliadas (SILVA et al., 2005). Para obtenção de qualidade satisfatória na produção de pisos, faz-se necessária a utilização de espécies e parâmetros de usinagem adequados, pois, segundo Silva et al. (2009), é preciso conhecer a estrutura da madeira e os parâmetros de usinagem para entender as relações que proporcionam bons resultados em qualidade.

Os principais parâmetros a serem considerados nas operações de usinagem são velocidade de avanço da madeira e velocidade de corte da ferramenta (MARTINS et al., 2013). A velocidade de avanço mede a velocidade com que a peça de madeira se desloca em direção à ferramenta de corte. A velocidade de corte é definida como o caminho percorrido pelo dente da ferramenta num determinado período de tempo, sendo expressa em metros por segundo. De acordo com Costes e Larricq (2002), a velocidade de corte pode interferir na qualidade da madeira usinada. A relação entre os dois parâmetros mencionados influencia diretamente a qualidade superficial da madeira. Silva et al. (2007) observam ainda que, ao se utilizarem baixos valores de velocidade de avanço e alta velocidade de corte, pode-se reduzir a qualidade da madeira usinada, ocasionando a carbonização da superfície da madeira. Altos valores de velocidade de avanço em relação à velocidade de corte podem ocasionar redução na qualidade da superfície usinada da madeira, havendo principalmente o arrancamento de fibras (SILVA et al., 2009).

O conceito de qualidade da superfície da madeira usinada é dividido por dois grupos de pesquisadores. O primeiro grupo leva em consideração a avaliação pelo avanço por dente (fz), expresso em marcas por polegada linear ou em milímetros; o segundo grupo considera não somente o fz, mas também a presença de falhas na superfície (SILVA, 2002). A presença de falhas na superfície da madeira pode ser avaliada por diferentes métodos, por exemplo, pela norma American Society for Testing and Materials D1666-11 (ASTM, 2011) e por rugosímetro de arraste.

Conforme Martins et al. (2013), as madeiras de Eucalyptus microcorys, E. cloeziana e Corymbia maculata podem ser utilizadas para pisos de acordo com resultados obtidos em ensaios físico-mecânicos. Porém é necessário analisar o comportamento da madeira de eucalipto em função de parâmetros da usinagem, como velocidade de avanço da madeira e velocidade de corte da ferramenta.

O objetivo do presente trabalho foi avaliar o comportamento das madeiras de Eucalyptus e Corymbia para produção de pisos, por meio de diferentes métodos de qualificação de superfícies, como também analisar a relação da densidade e desvio da grã com a rugosidade da madeira.

\section{MATERIAL E MÉTODOS}

Foram utilizadas cinco espécies (Tabela 1) provenientes do Projeto de Desenvolvimento e Pesquisa Florestal (PRODEPEF), implantado na Universidade Federal de Lavras (UFLA), em Lavras, MG.

Tabela 1. Relação das espécies utilizadas, por gênero.

Table 1. List of species used, by gender.

\begin{tabular}{ll}
\hline Gênero & Espécie \\
\hline \multirow{2}{*}{ Corymbia } & C. citriodora \\
& C. maculata \\
\hline \multirow{2}{*}{ Eucalyptus } & E. dunnii \\
& E. grandis \\
& E. urophylla \\
\hline
\end{tabular}

Após a derrubada das árvores, todas com 37 anos de idade, as toras foram desdobradas tangencialmente em tábuas por meio de serra de fita. Posteriormente foram entabicadas e secas até $12 \%$ de umidade em estufa convencional. A seguir, as tábuas secas foram transportadas e acondicionadas no Laboratório de Usinagem da Madeira (DCF/UFLA), onde foram confeccionados os corpos de prova, com dimensões de $800 \times 100 \times 25 \mathrm{~mm}$ $(\mathrm{C} \times \mathrm{L} \times \mathrm{E})$.

Os ensaios de usinagem se deram em plaina desempenadeira com três facas e sistema de exaustão. As velocidades de avanço (Vf) foram fixadas em 15 e $30 \mathrm{~m}^{*} \mathrm{~min}^{-1}$, utilizando para isso o inversor de frequência (W08). Foram utilizadas durante a usinagem as velocidades de corte (Vc) de 18 e $25 \mathrm{~m}^{*} \mathrm{~s}^{-1}$. A interação entre as duas velocidades de corte e as duas velocidades de avanço geraram quatro condições de usinagem: 1) Vf de $15 \mathrm{~m}^{*} \mathrm{~min}^{-1}$ e Vc de $18 \mathrm{~m}^{*} \mathrm{~s}^{-1}$; 2) Vf de $15 \mathrm{~m}^{*} \mathrm{~min}^{-1}$ e Vc de $25 \mathrm{~m}^{*} \mathrm{~s}^{-1}$; 3) Vf de $30 \mathrm{~m} * \mathrm{~min}^{-1}$ e Vc de $18 \mathrm{~m}^{*} \mathrm{~s}^{-1}$; 4) Vf de $30 \mathrm{~m} \mathrm{~min}^{-1}$ e Vc de $25 \mathrm{~m}^{*} \mathrm{~s}^{-1}$.

Para cada interação dos parâmetros de velocidade de avanço e de corte foram ensaiados cinco corpos de prova. Paralelamente, foi determinada em cada corpo de prova a densidade aparente, conforme o método 
descrito na NBR 7190 (ABNT, 1997), e o desvio da grã, de acordo com o método descrito por Silva (2002).

Após os ensaios de usinagem, foram realizadas as avaliações das superfícies, por meio da determinação do avanço por dente medido ( $\mathrm{f}_{\text {medido }}$ ), da análise visual (ASTM 1666-11, 2011) e da rugosidade $(\mathrm{Ra}$ e $\mathrm{Rz}$ ). Para tanto, foram demarcados retângulos de $10 \times 5 \mathrm{~mm}$ (C x L) e setas para indicar a localização e o sentido da leitura, respectivamente.

$\mathrm{Na}$ análise visual, os corpos de prova foram avaliados por três avaliadores isoladamente. Caso fosse observada divergência entre as notas dos avaliadores, seria realizada nova análise conjunta, buscando-se o consenso. Os corpos de prova foram classificados em 5 classes distintas (Tabela 2).

Tabela 2. Classes de qualidade da superfície de madeiras usinadas.

Table 2. Quality classes surface machined woods.

\begin{tabular}{lll}
\hline Nota & Classificação & Percentual de defeitos \\
\hline 1 & Excelente & $\mathrm{D}=0 \%$ (Sem defeitos) \\
2 & Bom & $0 \%<\mathrm{D}<50 \%$ \\
3 & Regular & $\mathrm{D}=50 \%$ \\
4 & Ruim & $50 \%<\mathrm{D}<100 \%$ \\
5 & Muito ruim & $\mathrm{D}=100 \%$ (Totalmente defeituosa) \\
\hline Fonte: Adaptação ASTM (2011)
\end{tabular}

Para análise estatística dos dados de densidade e desvio da grã, foi utilizado o delineamento inteiramente casualizado (DIC), com modelo estatístico conforme Equação 1. Para os dados de rugosidade (Ra e $\mathrm{Rz}$ ), o delineamento foi fatorial de $5 \times 2 \times 2$ (espécies x velocidade de avanço x velocidade de corte), com modelo estatístico conforme Equação 2. Nas comparações múltiplas de médias, foi utilizado o teste Scott-Knott, a $5 \%$ de significância.

$$
\begin{gathered}
Y_{i j}=\mu+\alpha_{i}+\varepsilon_{i j} \\
Y_{i j k l}=\mu+\alpha_{i}+\beta_{j}+\gamma_{k}+\alpha \beta_{i j}+\alpha \gamma_{i k}+\beta \gamma_{j k}+\alpha \beta \gamma_{i j k}+\varepsilon_{i j k l}
\end{gathered}
$$

em que: $Y_{i j}=$ valor observado na parcela experimental que recebeu o nível $i$ (i $=1,2$ e 3 ) do fator $\alpha$ e na repetição $k\left(\mathrm{k}=1,2\right.$ e 3); $Y_{i j k l}=$ valor observado na parcela experimental que recebeu o nível $i(\mathrm{i}=1,2$ e 3$)$ do fator $\alpha$, o nível $j(\mathrm{j}=1,2$ e 3) do fator $\beta$ e o nível $k(\mathrm{k}=1$ e 2) do fator $\gamma$ na repetição $1(1=1,2$ e 3); $\mu=$ representa uma constante geral; $\alpha_{i}=$ efeito do nível $i$ do fator $\alpha ; \beta_{j}=$ efeito do nível $j$ do fator $\beta$; $\gamma_{k}=$ efeito do nível $k$ do fator $\gamma ; \alpha \beta_{i j}=$ efeito da interação entre o nível $i$ do fator $\alpha$ e o nível $j$ do fator $\beta$; $\alpha \gamma_{i k}=$ efeito da interação entre o nível $i$ do fator $\alpha$ e o nível $k$ do fator $\gamma ; \beta \gamma_{j k}=$ efeito da interação entre o nível $j$ do fator $\beta$ e o nível $k$ do fator $\gamma ; \alpha \beta \gamma_{i j k}=$ efeito da interação entre o nível $i$ do fator $\alpha$, o nível $j$ do fator $\beta$ e o nível $k$ do fator $\gamma$; $\varepsilon_{i j}$ e $\varepsilon_{i j k l m}=$ erros experimentais.

Para avaliar as possíveis correlações existentes entre as propriedades de densidade aparente e desvio da grã com os parâmetros de rugosidade (Ra e Rz), empregou-se a matriz de correlação de Pearson a 5\% de significância.

\section{RESULTADOS}

\section{Avanço por dente medido}

A tabela 3 apresenta os valores médios de avanço por dente para os quatro tratamentos utilizados. Observa-se que os tratamentos não apresentaram o fz adequado para qualificar a superfície da madeira como boa segundo classificação proposta por SENAI (1995).

Tabela 3. Valores médios de avanço por dente medido para diferentes velocidades de avanço e velocidades de corte.

Table 3. Average values of feed per tooth measured for different speeds of advance and cutting speeds.

\begin{tabular}{llll}
\hline \multirow{2}{*}{ Condição de usinagem (tratamento) } & \multicolumn{2}{c}{ Velocidades } & \multirow{2}{*}{$\mathbf{f} \mathbf{z}_{\text {medido }}(\mathbf{m m})$} \\
\cline { 2 - 3 } & $\mathbf{d e}$ avanço $\left(\mathbf{m}^{*} \mathbf{m i n}^{\mathbf{- 1}}\right)$ & 18 & 4,9 \\
2 & 15 & 25 & 3,7 \\
2 & 15 & 18 & 10,0 \\
3 & 30 & 25 & 7,5 \\
\hline
\end{tabular}

FLORESTA, Curitiba, PR, v. 46, n. 3, p. 397 - 403, jul. / set. 2016.

Silva, F. A. V. da et al.

ISSN eletrônico 1982-4688

DOI: $10.5380 /$ rf.v46i3.43936 


\section{Avaliação conforme a norma ASTM D1666-11}

Como os resultados foram iguais entre as espécies, os defeitos foram analisados somente em função da velocidade de avanço da madeira e velocidade de corte da ferramenta. Na figura 1 podem ser visualizados os resultados da avaliação da qualidade da superfície da madeira conforme a norma ASTM D1666-11 (ASTM, 2011).

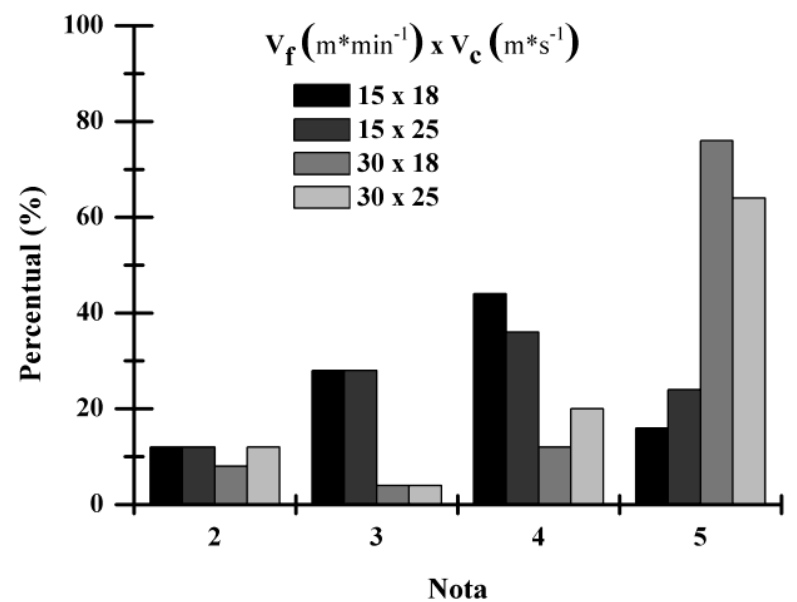

Figura 1. Média das notas segundo ASTM D1666-11(2011) entre as espécies para as diferentes velocidades de avanço (Vf) x velocidade de corte da ferramenta (Vc).

Figure 1. Average of notes according to ASTM D1666-11 (2011) between the species for different (Vf) $\mathrm{x}$ tool cutting speed $(\mathrm{Vc})$.

Os melhores resultados foram aqueles que receberam notas 2, porém os percentuais de notas foram baixos para todos os tratamentos, visto que os maiores índices de madeira foram classificados com as notas 4 e 5 .

\section{Parâmetros de rugosidade}

Os parâmetros de rugosidade Ra e Rz coletados foram submetidos à análise de variância (Tabela 4). A qualidade dos produtos de madeira está relacionada, entre outras características, à rugosidade superficial do produto (VEGA et al., 2007), sendo esta uma forma de avaliar a qualidade da superfície da madeira com maior precisão (MARTINS et al., 2011), embora tenha sido observado que todas as interações foram não significativas, em que apenas a espécie influenciou na variação do Ra e Rz.

Tabela 4. Resumo da análise de variância para os parâmetros de rugosidade "Ra" e "Rz" da superfície da madeira de Eucalyptus e Corymbia em diferentes padrões de usinagem.

Table 4. Summary of the analysis of variance for the roughness parameters "Ra" and "Rz" the surface of Eucalyptus and Corymbia in different patterns machining.

\begin{tabular}{|c|c|c|c|c|c|}
\hline \multirow{2}{*}{ Fonte de variação } & \multirow{2}{*}{ GL } & \multicolumn{4}{|c|}{ Quadrado médio } \\
\hline & & $\mathbf{R a}$ & & $\mathbf{R z}$ & \\
\hline Espécie (E) & 4 & 2,143 & & 80,046 & \\
\hline Velocidade avanço (Va) & 1 & 0,149 & ns & 1,947 & ns \\
\hline Velocidade de corte ( $\mathrm{Vc}$ ) & 1 & 0,810 & ns & 59,287 & ns \\
\hline $\mathrm{E} \times \mathrm{Va}$ & 4 & 0,752 & ns & 27,046 & $\mathrm{~ns}$ \\
\hline $\mathrm{E} \times \mathrm{Vc}$ & 4 & 0,498 & ns & 6,463 & ns \\
\hline $\mathrm{Vax} \mathrm{Vc}$ & 1 & 0,683 & ns & 2,035 & ns \\
\hline $\mathrm{E} \times \mathrm{Va} \times \mathrm{Vc}$ & 4 & 0,318 & ns & 22,497 & $\mathrm{~ns}$ \\
\hline Erro & 80 & 0,478 & & 23,798 & \\
\hline $\mathrm{CV}(\%)$ & & 27,92 & & 34,19 & \\
\hline
\end{tabular}

GL $=$ graus de liberdade $; \mathrm{CV}(\%)=$ coeficiente de variação experimental. ${ }^{\mathrm{ns}}=$ não significativo a $95 \%$ de probabilidade $;{ }^{*}=$ significativo a 95\% de probabilidade.

Os valores significativos para os parâmetros de rugosidade Ra e Rz entre espécies são devidos às diferentes densidades e aos diferentes valores encontrados para o ângulo da grã (Tabela 5). 
Tabela 5. Resumo da análise de variância para os valores de densidade aparente e desvio da grã das madeiras de Eucalyptus e Corymbia.

Table 5. Summary of the analysis of variance for the values of bulk density and deviation of the grain of Eucalyptus and Corymbia wood.

\begin{tabular}{lccc}
\hline \multirow{2}{*}{ Fonte de variação } & \multirow{2}{*}{ GL } & \multicolumn{2}{c}{ Quadrado médio } \\
\cline { 3 - 4 } & & Densidade aparente & Desvio da grã \\
\hline Espécie & 4 & $0,0836^{*}$ & $16,184^{*}$ \\
Erro & 20 & 0,0024 & 6,144 \\
CV $(\%)$ & & 5,06 & 73,92 \\
\hline
\end{tabular}

$\overline{\mathrm{GL}}=$ graus de liberdade; $\mathrm{CV}(\%)=$ coeficiente de variação experimental; ${ }^{\mathrm{ns}}=$ não significativo a $95 \%$ de probabilidade; ${ }^{*}=$ significativo a $95 \%$ de probabilidade. estudadas.

A tabela 6 apresenta os resultados de densidade aparente e de desvio de grã para as cinco espécies

Tabela 6. Densidade aparente a $15 \%$ de umidade e o desvio da grã das cinco espécies.

Table 6. Bulk density at $15 \%$ moisture and the deviation of the grain of the five species.

\begin{tabular}{cccccc}
\hline \multirow{2}{*}{ Característica } & C. citriodora & C. maculata & E. dunnii & E. grandis & E. urophylla \\
\cline { 2 - 6 } & $1,036 \mathrm{~A}$ & $0,979 \mathrm{~B}$ & $0,927 \mathrm{C}$ & $0,871 \mathrm{D}$ & $0,998 \mathrm{~B}$ \\
Densidade aparente & $3,033 \mathrm{~B}$ & $2,767 \mathrm{~B}$ & $3,533 \mathrm{~B}$ & $4,833 \mathrm{~A}$ & $2,600 \mathrm{~B}$ \\
Desvio da grã &
\end{tabular}

Médias seguidas pela mesma letra na mesma linha não diferem entre si pelo teste de Tukey a 5\% de significância.

Os valores médios dos parâmetros de rugosidade Ra e Rz podem ser visualizados na tabela 7. Observase pouca variação de rugosidade entre os diferentes parâmetros de usinagem.

Tabela 7. Valores médios dos parâmetros de rugosidade $\mathrm{Ra}$ e $\mathrm{Rz}$ para diferentes velocidades de avanço e velocidade de corte.

Table 7. Average values of Ra roughness parameters and $\mathrm{Rz}$ for different speeds of advance and cutting speed.

\begin{tabular}{lcc}
\hline Velocidade de avanço $\left(\mathbf{m}^{*} \mathbf{m i n}^{-\mathbf{1}}\right) \mathbf{x}$ Velocidade de corte $\left(\mathbf{m}^{*} \mathbf{s}^{-\mathbf{1}}\right)$ & $\mathbf{R a}(\boldsymbol{\mu m})$ & $\mathbf{R z}(\boldsymbol{\mu m})$ \\
\hline 15 x 18 & 2,44 & 15,04 \\
15 × 25 & 2,43 & 13,78 \\
30 x 18 & 2,69 & 15,04 \\
30 x 25 & 2,34 & 13,22 \\
\hline
\end{tabular}

\section{DISCUSSÃO}

\section{Avanço por dente medido}

Os valores de $\mathrm{fz}$ medidos estão associados principalmente aos parâmetros de usinagem, podendo ser observado no trabalho de Silva et al. (2009), cujos autores encontraram aumento do $\mathrm{fz}_{\text {medido }}$ com o acréscimo da velocidade avanço, sendo estudada a madeira de Eucalyptus sp. aos 32 anos. Os valores de $\mathrm{fz}_{\text {medido }}$ foram 1,4; 3,1 e 6,3 para as velocidades de avanço de 6,15 e $30 \mathrm{~m}^{*} \mathrm{~min}^{-1}$, respectivamente.

Carvalho et al. (2010) utilizaram velocidade de avanço de $18 \mathrm{~m}^{*} \mathrm{~min}^{-1}$ e rotação de $2800 \mathrm{~min}^{-1}$ para a madeira de Khaya ivorensis com idade em torno de 10 anos e obtiveram valor de fz de 3,46, inferior ao do tratamento 2 do presente trabalho. Isso pode ter sido ocasionado pela menor rotação ou pela menor densidade aparente da madeira de $K$. ivorensis utilizada por Carvalho et al. (2010). O mesmo foi observado no trabalho de Dias Junior et al. (2013), que encontraram $\mathrm{fz}_{\text {medido }}$ de 2,5 para velocidade de avanço de $13 \mathrm{~m}^{*} \mathrm{~min}^{-1}$ e rotação de $2600 \mathrm{~min}^{-1}$ para quatro espécies de Eucalyptus com 20 anos. As densidades aparentes desses eucaliptos foram altas, como no presente trabalho.

De acordo com Weinig (2000), o avanço por dente indicado para pisos de madeira é de 1,5 mm. Valores maiores que 1,5 mm são indicados para fabricação de produtos como corrimão, peças estruturais e esquadrias para construção civil, entre outros. Considerando os valores médios, nenhuma condição de usinagem utilizada ofereceu superfície adequada para confecção de piso de madeira. Nesse caso, as madeiras requerem o retrabalho, como, por exemplo, lixamento. De acordo com SENAI (1995), os valores médios obtidos para os avanços por dente caracterizam a superfície como de qualidade de acabamento grosseiro. 


\section{Avaliação conforme a norma ASTM D1666-11}

Ao se avaliar a qualidade da madeira segundo a norma ASTM D1666-11 (ASTM, 2011) entre as diferentes espécies, foi constatada a mesma quantidade de defeitos entre elas, diferentemente do trabalho de Souza et al. (2009), em que foi obtida melhor qualidade da superfície na madeira de Eucalyptus urophylla aos 6 anos, em comparação com Eucalyptus camaldulensis aos 10 anos e Eucalyptus urophylla aos 8 anos do mesmo trabalho.

Os tratamentos com velocidade de avanço de $15 \mathrm{~m}^{*} \mathrm{~min}^{-1}$ foram os que apresentaram menor número de peças de madeira que receberam notas 5, classificadas como muito ruim (Figura 1). Zamarian et al. (2012) afirmam que a maior velocidade de avanço da peça resultará em uma superfície da madeira de pior qualidade.

\section{Parâmetros de rugosidade}

Kilic et al. (2006) afirmam que os parâmetros de usinagem também são responsáveis pela rugosidade da madeira. Maiores velocidades de avanço resultam em superfícies com maior rugosidade (AGUILERA; ZAMORA, 2007). Isso não foi constatado no presente trabalho. Braga et al. (2014) também estudaram diferentes velocidades de avanço para madeira de Coffea arabica e não encontraram influência desse parâmetro na rugosidade da madeira.

Os resultados do presente trabalho diferem dos encontrados por Dias Junior et al. (2013), os quais trabalharam com madeiras mais jovens de quatro espécies de Eucalyptus e de menor densidade, observando que nas maiores velocidades de avanço os valores de Ra e Rz foram maiores, e para baixas velocidades de avanço com rotações maiores tem-se uma diminuição nos valores de rugosidade.

Aguilera e Muñoz (2011), em trabalho com Acacia melanoxylon (acácia-da-austrália) e Sequoia sempervirens (sequoia), observaram menores valores de rugosidade para madeira com maior densidade, o que resultou em maior qualidade superficial.

Com base nos gráficos gerados na avaliação da rugosidade, foi observado que no perfil da superfície aplainada da madeira de maior densidade houve menores picos e vales profundos, bem como menor extensão destes. Por outro lado, o perfil da superfície da espécie com menor densidade foi caracterizado por uma incidência de maiores picos e vales.

Os valores médios de rugosidade Ra nos diferentes parâmetros de usinagem foram próximos do trabalho realizado pelos autores Lopes et al. (2014), que avaliaram a superfície da madeira de E. grandis, E. urophylla e E. dunnii aos 18 anos utilizando velocidade de avanço de $15 \mathrm{~m}^{*} \mathrm{~min}^{-1}$ e rotação de $3450 \mathrm{~min}^{-1}$, obtendo a rugosidade Ra de 2,27.

Ao verificar os resultados obtidos na avaliação visual da superfície da madeira de acordo com a norma ASTM D1666-11 (ASTM, 2011) e os resultados de rugosidade, observa-se distinção entre eles, pois, na velocidade de avanço de $30 \mathrm{~m}^{*} \mathrm{~min}^{-1}$ e velocidade de corte de 18 e $25 \mathrm{~m}^{*} \mathrm{~s}^{-1}$, foram obtidas mais peças qualificadas como muito ruins conforme a norma, enquanto na rugosidade os resultados foram semelhantes em todos os parâmetros de usinagem. Por meio desses resultados, observou-se que é preciso o uso de mais de um método de avaliação quando se trata de qualificação de superfície usinada.

\section{CONCLUSÕES}

- O maior índice de tábuas das duas espécies foi classificado como ruim (nota 4) e muito ruim (nota 5), com base na ASTM D1666-11 (ASTM, 2011).

- Os valores obtidos para o avanço por dente para todas as velocidades de avanço e velocidade de corte foram acima dos indicados para pisos de madeira.

- Os valores dos parâmetros de rugosidade Ra e Rz não foram influenciados pelas diferentes velocidades de avanço e velocidade de corte.

- Fatores como densidade e desvio de grã influenciam diretamente a rugosidade da madeira usinada.

- Os menores valores dos parâmetros de rugosidade Ra e Rz foram obtidos para as madeiras com maior densidade, pois, quanto menor a densidade e maior o ângulo da grã, maior foi o parâmetro de rugosidade Ra e Rz.

- Ao se utilizar os diferentes métodos de qualificação de superfícies nas madeiras de Eucalyptus e Corymbia, observou-se baixo potencial para utilizar essas madeiras em pisos. No entanto, é necessário que sejam realizados outros estudos, com diferentes velocidades de avanço e de corte, para verificar a possibilidade de melhoria nas qualidades superficiais dessas madeiras.

\section{REFERÊNCIAS}

AGUILERA, A.; MUNOZ, H. Rugosidad superficial y potencia de corte en el cepillado de Acacia melanoxylon y Sequoia sempervirens. Maderas, Cienc. Tecnol., v. 13, n. 1, p. 19-28, 2011. 
AGUILERA, A.; ZAMORA, R. Monitoreo del proceso de maquinado de aromo australiano (Acacia melanoxylon) con emisión acústica y su relación con la calidad superficial resultante. Maderas, Cienc. Tecnol., v. 9, n. 3, p. 323-332, 2007.

AMERICAN SOCIETY FOR TESTING AND MATERIALS (ASTM). ASTM 1666- 11: standard test methods for conducting machining tests of wood and wood-base panel materials. Philaldelphia. p. 226$245,2011$.

ASSOCIAÇÃO BRASILEIRA DE NORMAS TÉCNICAS (ABNT). NBR 7190: Projeto de Estruturas de Madeira - Rio de Janeiro, 1997.

ASSOCIAÇÃO NACIONAL DOS PRODUTORES DE PISOS DE MADEIRA (ANPM). Disponível em: <http://www.anpm.org.br>. Acesso em: 10/04/2015.

BRAGA, P. P. C.; SILVA, J. R. M.; NÉRI, A. C. Qualidade da superfície de madeira de Coffea arabica. Cerne, v. 20, n. 1, p. 21-28, 2014.

CARVAlHO, A. M. de; SILVA.; B. T. B. da; LATORRACA, J. V. F. de. Avaliação da usinagem e caracterização das propriedades físicas da madeira de mogno africano (Khaya ivorensis a. Chev.). Cerne, Lavras, v. 16, p. 106-114, 2010.

COSTES, J.; LARRICQ, P. Towards high cutting speed in wood milling. Annals of Forest Science, v. 59, p. 857-865, 2002.

DiAS JUNiOR, A. F.; SANTOS, P. V.; PACE, J. H. C.; CARVAlHO, A. M.; LATORRACA, J. V. F. de. Caracterização da madeira de quatro espécies florestais para uso em movelaria. Ciência da Madeira (Braz. J. Wood Sci.), Pelotas, v. 4, n. 1, p. 93-107, 2013.

KILIC, M.; HIZIROGLU, S.; BURDURLU, E. Effect of machining on surface roughness of wood. Building and Environment, v. 41, n. 8, p. 1074-1078, 2006.

LOPES, C. S. D.; NOLASCO, A. M.; TOMAZELLO FILHO, M.; DIAS, T. S. Avaliação da rugosidade superficial da madeira de Eucalyptus sp. submetida ao fresamento periférico. Cerne, v. 20, n. 3, p. 471-476, 2014.

MARTINS, M.; SILVA, J. R. M.; LIMA, J. T.; GONÇALVES, M. T. T.; FILIPE, A. P. Simulação em uso dos pisos de madeira de Eucalyptus sp. e Corymbia maculata. Cerne, Lavras, v. 19, n. 1, p. 151-156, 2013.

MARTINS, S. A.; FERRAZ, J. M.; SANTOS, C. M. T.; MENEZZI, C. H. S. D.; SOUZA, M. R. Efeito da usinagem na rugosidade da superfície da madeira de Eucalyptus benthamii. Floram, v. 18, n. 2, p. 135-143, 2011.

SERVIÇO NACIONAL DE APRENDIZAGEM INDUSTRIAL (SENAI). Acabador de móveis. Ubá: CFP/JAGS, 1995. 29 p.

SILVA, J. R. M. Relações da usinabilidade e aderência do verniz com as propriedades fundamentais do Eucalyptus grandis Hill Ex. Maiden. 2002. 179 p. Tese (Doutorado em Engenharia Florestal) - Universidade Federal do Paraná, Curitiba, 2002.

SILVA, J. R. M.; LIMA, J. T.; TRUGILHO, P. F. Usinabilidade da madeira de Eucalyptus grandis em diferentes regiões da medula à casca. Cerne, v. 13, n. 1, p. 25-31, 2007.

SILVA, J. R. M; MARTINS, M.; OLIVEIRA, G. M. V.; BRAGA, P. P. B. Parâmetros de qualidade da usinagem para determinação dos diferentes usos da madeira de Eucalyptus. Cerne, Lavras, v. 15, n. 1, p. 75-83, 2009.

SILVA, J. R. M.; MUNIZ, G. I. B.; LIMA, J. T.; BONDUELLE, A. F. Influência da morfologia das fibras na usinabilidade da madeira de Eucalyptus grandis Hill ex. Maiden. Revista Árvore, v. 29, n. 3, p. 479-487, 2005.

SOUZA, M. O. A.; SIlVA, J. C.; LUCIA, R. M. D.; EVANGEliSTA, W. V. Avaliação da madeira de Eucalyptus camaldulensis e Eucalyptus urophylla em ensaios de usinagem, visando à produção moveleira. Revista Árvore, Viçosa, v. 33, n. 4, p. 751-758, 2009.

VEGA, M.; AGUILERA, A.; MEAUSOONE, P. J. Esfuerzos de corte y rugosidad superficial en el fresado de pino radiata y su relación con emisión acústica. Maderas, Cienc. Tecnol., v. 9, n. 2, pp. 161-169, 2007.

WEINIG. Obserflächenqualität. Werkzeug, Präsentation Hydrotechnik, Jointen. Author: Andreas Schreck. V. cd. 2000 .

ZAMARIAN, E. H. C.; ALBUQUERQUE, C. E. E.; MATOS, J. L. M. Usinagem da madeira de bracatinga para uso na indústria moveleira. Floresta, v. 42, n. 3, p. 631-638, 2012. 